\title{
Assessment of heat provocation tests on the human gingiva: the effect of periodontal disease and smoking
}

\author{
E Molnár, Z Lohinai, A Demeter, B Mikecs, Z Tóth, J Vág
}

Department of Conservative Dentistry, Faculty of Dentistry, Semmelweis University, Budapest, Hungary

Received: September 10, 2014

Accepted after revision: February 9, 2015

\begin{abstract}
Periodontal inflammation is associated with morphological changes in the blood vessels which may influence the regulation of gingival blood flow $(\mathrm{GBF})$. Our aim was to adapt the heat provocation test to the human gingiva to assess vascular reactivity in periodontal inflammation. Method: GBF was recorded by Laser Doppler Flowmetry before and after heat provocation in healthy volunteers $(n=50)$. Heat was generated either by warm saline or a halogen lamp. The latter method was also utilized for a heat test in non-smoking and smoking patients with periodontal inflammation. The circulatory parameters were correlated to the inflammatory marker, i.e. gingival crevicular fluid (GCF) production measured by Periotron. Results: Local application of heat caused a rapid, significant and transient increase in GBF regardless of the method used. The increase in the speed and not in the concentration of moving blood cells was responsible for increased GBF. Higher GCF values were correlated with increased peak flow, flux pulse amplitude and faster restoration of GBF after the test in non-smokers, but not in smokers. Conclusions: The heat test could be a valuable tool to check the vascular reactivity of gingival vessels. Moderate periodontal inflammation may facilitate gingival vascular responsiveness which can be suppressed by smoking.
\end{abstract}

Keywords: blood flow, Laser Doppler Flowmetry, crevicular fluid, smoking, periodontitis, gingivitis, heat test

Periodontitis is an inflammatory disease of tooth supporting tissues. It affects approximately $75 \%$ of the population. Severe periodontitis was identified as the $6^{\text {th }}$ most prevalent condition in the Global Burden of Disease Study 2010 coordinated by the Institute for Health Metrics and Evaluation, affecting $11 \%$ of the global population (32). Twenty years ago over $95 \%$ of the Hungarian population was reported to have some kind of periodontal disease (5), and five years ago this figure was still as high as $88 \%$ (14). Therefore, periodontal disease is a major burden on the society in Hungary. For this reason, and because periodontal disease has been associated with an increased risk of major systemic diseases, such as coronary heart disease, stroke, diabetes and osteoporosis $(18,30)$, there is an urgent need for improving the treatment of periodontitis.

When the gingiva is inflamed dental biofilms release bioactive substances (51), which induce a host response in the gingiva and in periodontal tissues. Vascular changes and remodelling in periodontitis were reported to affect the progression of the disease $(61,62)$. Some vascular changes including the dilation of vessels, the formation of tortuous looping structures and the development of high endothelial cells could promote the defence mechanism against bacteria, however, the formation of perivascular hyaline material and the accumulation of basement membrane rests may assist the progression of periodontitis (61). Interestingly,

Corresponding author: Dr János Vág

Department of Conservative Dentistry, Semmelweis University

Szentkirályi utca 47, H-1088 Budapest, Hungary

Phone: +36-1-317-15-98; Fax: +36-1-317-11-22; E-mail: drvagjanos@gmail.com 
the thickening of the basal lamina around microvessels occurs just as in the case of diabetes mellitus (DM) $(56,59)$. In DM patients, microvascular complications in other organs, e.g. neuropathy, nephropathy and retinopathy, were found to be associated with the presence of a more severe inflammatory pathology of periodontal tissues (49). A recent study (53) found decreased post-occlusive reactive hyperaemia in the gingiva of a diabetic rat compared to a healthy one and this response was further reduced by experimentally induced periodontitis. However, there is no direct evidence for the impairment of vascular reactivity in the human gingiva in periodontitis and/or DM.

Similar to DM, smoking has a negative impact on periodontal status (21). Smoking has distinct acute and chronic vascular effects $(6,34,38)$, but no data are available on the effect of smoking on vascular reactivity in periodontitis.

In order to investigate vasoregulatory changes in periodontitis or in systemic conditions such as DM or smoking, a vascular reactivity test has to be elaborated for the human gingiva. Vascular reactivity could be assessed by various provocation tests including iontophoresis of vasoactive molecules, post-occlusive hyperaemia or heat challenge $(28,48)$. It should be possible to apply the test easily and non-invasively in the oral cavity without any discomfort to the patient, furthermore, it should be short enough to use it routinely for high-throughput clinical investigations. As surgical intervention is a common treatment in periodontitis, the test should not interfere with wound healing. Among vascular reactivity tests the heat provocation test seems to be an appropriate method meeting these requirements.

The aim of the present study is to set up a heat provocation test applicable in clinical practice in terms of application technique, reversibility and time. In the second part of the study heat tests are evaluated on subjects with a gingiva affected by inflammation of varying severity. In our experiment, subjects were divided into smoking and non-smoking groups to explore the possible interaction of smoking and inflammation with regard to the microcirculation of the gingiva.

\section{Materials and Methods}

This study was performed on randomly chosen, systematically healthy incoming patients of the Department of Conservative Dentistry at Semmelweis University, Budapest, Hungary. Exclusion criteria were pregnancy, general diseases, long-term medication (except for contraceptives) and the use of antibacterial or anti-inflammatory medication. All participants $(n=50)$ were provided with a complete explanation of the procedures allowing them to give an informed consent. The study was performed in accordance with the Helsinki Declarations and was approved by the Regional and Institutional Ethics Committee (034310/2014/OTIG). All patients agreed to participate in the study and gave their written informed consent.

Blood flow to the gingival margin (GBF) was measured by Laser Doppler Flowmetry (780 nm; MoorLAB; Moor Instruments Ltd, Devon, UK). Laser Doppler instruments measure net red blood cell flux (Flux): the product of the average speed of the blood cells (Speed) and the concentration of moving red blood cells $(\mathrm{CMBC})$. Blood perfusion readings were made at rest in a room with steady ambient temperature $\left(26^{\circ} \mathrm{C}\right)$. Subjects were forbidden to brush their teeth, gargle or eat and drink anything for 30 minutes prior to the measurements. Each patient was placed comfortably in supine position in a dental chair and was left undisturbed for a minimum of 15 minutes before any measurements were taken. The lips were retracted with a set of cheek retractors. Care was taken to ensure that the mucosal surface adjacent to the site of recording remained unstrained. A straight laser Doppler probe (outer diameter: 
$1.5 \mathrm{~mm}$; Moor Instruments Ltd, UK) was attached to the flowmeter and directed $1 \mathrm{~mm}$ apical to the mid-buccal gingival margin at perpendicular angle, without touching the gingiva. The probe was positioned using a steel manipulator anchored in a custom-made silicone occlusion block. The laser Doppler flowmeter was connected to a computer and the readings were recorded by data acquisition software (MoorSoftMoorLab v2.01, Moor Instruments Ltd, Devon, UK). Blood perfusion was recorded with a sampling rate of 40 measurements per second and averaged by seconds.

Systemic blood pressure (systolic and diastolic) and the heart rate were recorded by an automated blood pressure monitor (Omron M4; Omron Healthcare Inc., Kyoto, Japan). In all experiments the blood pressure was measured before and after the blood flow recordings and the mean arterial blood pressure (MAP) was calculated.

Gingival crevicular fluid (GCF) inflammatory marker production was measured using a crevice fluid meter (Periotron 8000; Ora Flow Inc., NY, USA) by means of measuring the change in the electrical capacitance of filter paper strips (Periopaper; Ora Flow Inc., NY, USA), expressed in Periotron units (PU). The Periopaper was gently inserted into the gingival sulcus for 10 seconds to collect extravasation.

\section{The effect of warm saline on GBF in the healthy gingiva}

This experiment was performed on nine non-smoking volunteers with a healthy gingiva. Blood flow was recorded before, during (30 s) and after dropping $2 \mathrm{ml}$ of pre-warmed $\left(44^{\circ} \mathrm{C}\right)$ sterile saline solution on the marginal gingiva right next to the laser Doppler probe. Baseline GBF was recorded for 1 minute. The recording of gingival perfusion was continued for an additional 5 minutes after carrying out the test.

\section{The effect of light-induced heat on GBF in the healthy gingiva}

This experiment was performed on twelve non-smoking volunteers with healthy gingiva. Heat was generated on the gingiva using a dental curing light (IvoclarVivadent AG, Liechtenstein, $35 \mathrm{~W}$ ) from which the light filter was removed. The light guide was directed to the marginal gingiva at a distance of $1.5 \mathrm{~cm}$. GBF was recorded before and 5 minutes after heat provocation which was applied for 80 seconds on the marginal gingiva around the laser Doppler probe. GBF and blood pressure were recorded in a similar way as in the first set of experiments with the exception that GBF could not be recorded during the application of light as it interfered with the laser Doppler measuring signal.

\section{The effect of periodontal inflammation on heat-induced hyperaemia in non-smokers and smokers}

This group was composed of twenty-nine volunteers with a periodontal condition of varying severity, from healthy to suffering from a moderately severe inflammation assessed by a GCF reading (0-71). These patients were also systemically healthy based on the same exclusion criteria as above and separated into two groups: smokers $(n=11)$ and non-smokers $(n=18)$. Prior to blood flow measurements GCF production and blood pressure were measured as above. As in the previous set of experiments, GBF was recorded for at least 1 minute before and 5 minutes after the application of heat induced by light. The following circulatory parameters were calculated to characterize the individual heat response curve: maximum absolute change (MAX), maximum percentage change from baseline (MAX\%), the time to decrease to one third of the MAX\% corresponding to the speed of recovery after hyperaemia 
(RT, chosen due to differences in the position where the end of the curve returns) and the area under the curve from the start of recording after heat stimulation to the point of RT (Area). Average gingival flux pulse amplitude (GFPA) was also calculated at baseline (GFPA-bsl) and the first 15 seconds after heat provocation had been completed (GFPA-heat).

\section{Statistics}

For data with normal distribution (MAP, baseline GBF, MAX, MAX\%, GFPA) the mean and \pm standard error of the mean (SEM), the median and the interquartile range for non-normal distribution (age, GCF, RT, Area) were calculated. Alterations in the circulatory parameters as a function of time (repeated measurement factor) were statistically evaluated by the analysis of variance (ANOVA). The circulatory values of various periods were compared to the baseline period by Dunnett's test.

The parameters of smoking and non-smoking groups were compared either by parametric T-test (MAP, MAX, MAX\%, GFPA) or by non-parametric Kruskall-Wallis test (age, GCF, baseline GBF, RT, Area), depending on the distribution of the data and homogeneity of variances (Levene's test). The relationship between MAP and GBF was examined calculating the Pearson correlation coefficient. For the correlation between age, GCF, MAX, MAX\%, RT, Area and GFPA the Spearman test was used.

An alpha value of $p<0.05$ was used for all statistical analyses. Statistical evaluation was carried out using statistics software (Statistica; StatSoft Inc., Tulsa, OK, USA).

\section{Results}

\section{The effect of warm saline on GBF in the healthy gingiva}

The average MAP of this group of patients was $107 \pm 4 \mathrm{mmHg}$. The Flux value represents GBF and in our experiments we recorded the components of the flux (CMBC and Speed) separately as well in order to better characterize the vascular changes in the gingiva after the heat challenge. The application of warm saline to the buccal gingiva resulted in a quick increase in CMBC for periods of $20 \mathrm{~s}$ (Fig. 1). These values within this period were very noisy, therefore we excluded from the statistical analysis as they suggest an artefact due to the reflection of the laser light from the surface of the saline flow and/or mechanical irritation of the dropping. At the end of heat provocation CMBC dropped to the baseline level and remained there while the Speed and the Flux increased rapidly in parallel. Flux reached its peak response $(76 \pm 6.0 \%) 21$ seconds after completing the application of heat. The percentage mean changes during saline application; the mean values for every minute after application are shown in Table I. Increased GBF after heat application was due solely to the increase in the average speed of blood cells without any change in CMBC. Therefore, in further experiments only the Flux was used to estimate GBF.

\section{The effect of light-induced heat on GBF in healthy gingiva}

The average MAP of this group of patients was $109 \pm 5 \mathrm{mmHg}$, and it did not differ statistically from the warm saline group. The recording of GBF (Flux values) was started just after the halogen light had been switched off as it interfered with laser Doppler measurements. The changes in GBF after the application of light were expressed as a percentage of the baseline and the mean change is shown in Fig. 2. 


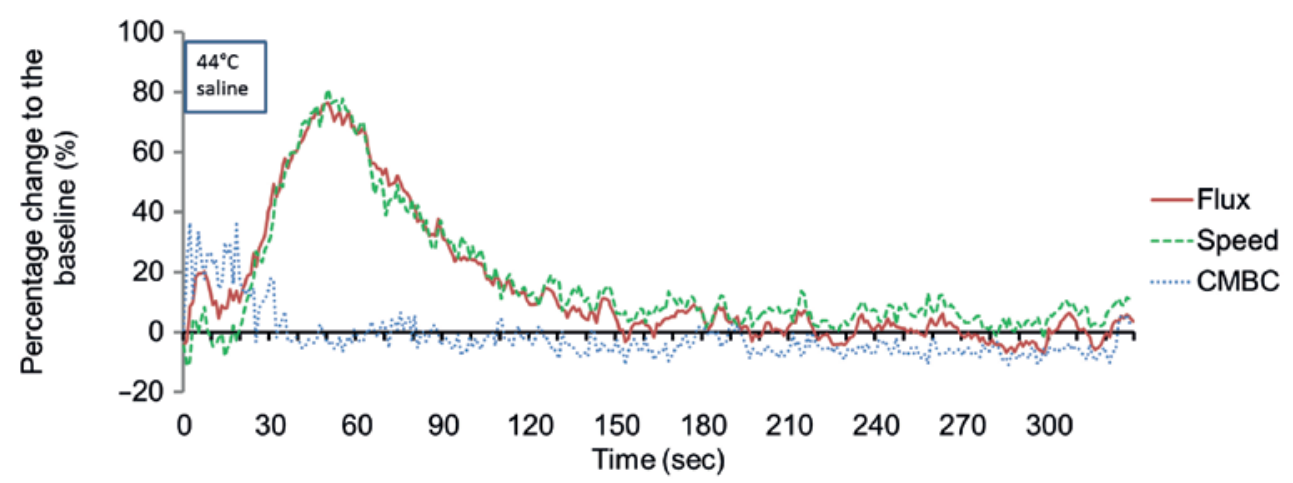

Fig. 1. The percentage change of the flux (gingival blood flow), the speed of the moving blood cells and the concentration of moving blood cells (CMBC) compared to the baseline during and after local application of warm saline. The Flux value represents the blood flow in the gingival margin as calculated by the multiplication of $\mathrm{CMBC}$ in the measured tissue volume and the average velocity of the cells (Speed). The mean values indicated on the graph were calculated from nine individual experiments where 40 values were recorded per seconds and were averaged for $1 \mathrm{~s}$. The period of saline application corresponds to the width of the framed text $\left(44^{\circ} \mathrm{C}\right.$ saline). Note that the $\mathrm{CMBC}$ values within this period are very noisy possibly due to the reflection of the laser light from the surface of the saline flow and/or mechanical irritation of the dropping

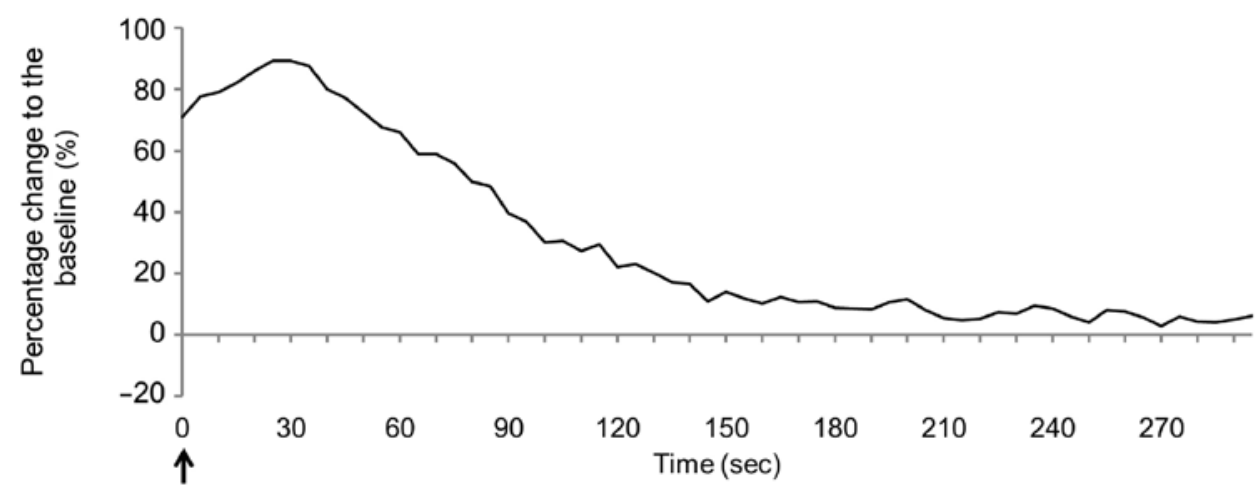

Fig. 2. The effect of light-induced heat on the mean Flux (gingival blood flow) in the gingiva of healthy subjects. The mean values from twelve individual experiments on the graph were calculated after the 40 values recorded per seconds were averaged for $1 \mathrm{~s}$. The arrow at 0 indicates the end of light application. The recording of the Flux started just after the halogen light was switched off as it interfered with laser Doppler measurements

Table I. Percentage mean changes of blood perfusion parameters compared to the baseline measured by a laser Doppler instrument after warm saline treatment (mean $\pm \mathrm{SEM})$

\begin{tabular}{|l|c|c|c|c|c|}
\hline & \multicolumn{5}{|c|}{ Time of measurement (\%) } \\
\hline$n=9$ & Min 1 & Min 2 & Min 3 & Min 4 & Min 5 \\
\hline GBF (Flux) & $56 \pm 4.8^{* * *}$ & $14 \pm 8.5$ & $3 \pm 4.3$ & $1 \pm 2.9$ & $-1 \pm 3.6$ \\
\hline CMBC & $0 \pm 3.8$ & $-3 \pm 2.3$ & $-4 \pm 1.5$ & $-6 \pm 3.5$ & $-6 \pm 3.4$ \\
\hline Speed & $55 \pm 4.7 * * *$ & $17 \pm 7.1$ & $7 \pm 3.3$ & $6 \pm 2.5$ & $5 \pm 2.5$ \\
\hline
\end{tabular}

GBF(Flux): gingival blood flow as measured by net red blood cell flux (Flux); Speed: average speed of the blood cells; CMBC: the concentration of moving red blood cells; $* * * p<0.001$ from the respective baseline values 
The GBF values for each minute recoding were averaged and tested for statistical differences to the baseline values. The value of averaged GBF was significantly higher at minutes 1 and $2(80 \pm 12 \%, p<0.001$ and $44 \pm 10, p<0.001$, respectively). After 2 minutes GBF returned to baseline values (min 3: $15 \pm 5 \%$, NS; $\min 4: 8 \pm 4 \%$, NS; $\min 5: 7 \pm 5 \%$, NS). The mean peak GBF value was $89 \pm 15 \%$ at $30 \mathrm{~s}$ after heat application had been finished which is very close to the value obtained in the first experiment. The average RT time was $110 \mathrm{~s}$.

Both methods were effective in inducing a rapid increase in GBF and even after provocation was finished GBF remained at an increased level long enough for data acquisition. This was an important criterion as both methods interfered with laser Doppler measurements, hindering recording during the provocation test. The application of warm saline was technically more demanding and sometimes run-off fluid caused discomfort to the patient resulting in movement artefacts. Therefore, in further experiments we opted for heat-induced light for the heat test.

\section{The effect of periodontal inflammation and smoking on heat-induced hyperaemia}

There were no significant differences observed between the non-smoking and the smoking group in terms of most of the baseline values such as age (26 (24-41) years vs 25 (24-28) years), MAP (116 \pm 4.2 vs $113 \pm 3.8 \mathrm{mmHg})$, GCF (10 (5-24) PU vs 3 (2-10) PU), GBF-bsl (172 (143-312) Flux vs 213 (166-238) Flux), MAX (415 \pm 41 Flux vs $450 \pm 52$ Flux), MAX\% (101 $\pm 12 \%$ vs $112 \pm 15 \%)$ and Area (10 (6-17) vs $14(11-18))$. On the other hand, there was a significant difference in RT values between the two groups (85 s (55-105) vs 115 $\mathrm{s}(75-155), p<0.05)$. GFPA was $68 \pm 7$ Flux at baseline and increased to $114 \pm 10$ Flux $(p<$ $0.001)$ after heat provocation in non-smokers and similarly changed from $79 \pm 9$ Flux to 117 \pm 12 Flux $(p<0.001)$ in smokers. Smoking itself did not influence absolute GFPA values. No change was observed in relative GFPA values (GFPA-heat/GFPA-bsl) after the application of heat in the non-smoking group ( $40 \pm 3 \%$ vs $40 \pm 3 \%$ ), but in the smoking group relative GFPA decreased significantly after the heat test ( $44 \pm 5 \%$ vs $37 \pm 4 \%, p<0.05$ ), indicating a significant $(p<0.05)$ interaction between the effect of smoking and heat test.

There was no correlation observed between the GBF values measured before heat provocation (baseline GBF) and the MAP of patients in either the non-smoking or the smoking group ( $r=0.13$, NS and $r=-0.44$, NS, respectively). As there was no change in MAP before and after heat provocation, the GBF values were used for further comparison instead of vascular resistance or conductance. The correlations of circulatory parameters to age or GCF are shown both for the non-smoking and the smoking group in Table II. No significant correlation was observed to age in any groups. No correlation was found between GCF and baseline GBF but a moderate positive correlation was found between GCF and the absolute value of the maximum increase in GBF (MAX) due to heat in the non-smoking group only and not in the smoking group. Similarly, GFPA was highly correlated to GCF values both in the baseline period (GFPA-bsl) and after heating (GFPA-heat) only in the nonsmoking group. No correlation was observed between GCF and relative GFPA in either period or group (data not shown). A strong negative correlation was found between GCF and RT $(r=-0.64, p<0.01)$ in non-smokers, but the same correlation was not found in the smoking group. 
Table II. Correlation coefficients of age and GCF to the GBF parameters in non-smokers and smokers

\begin{tabular}{|l|l|c|c|c|c|c|c|c|}
\hline & & bsl & MAX & MAX\% & RT & Area & Bsl-GFPA & Heat-GFPA \\
\hline Non-smokers & age & 0.13 & -0.02 & -0.27 & -0.28 & -0.43 & 0.32 & 0.04 \\
\hline & GCF & 0.44 & $0.48^{\#}$ & -0.05 & $-0.64 \# \#$ & -0.43 & $0.65^{\# \#}$ & $0.69 \# \#$ \\
\hline Smokers & age & -0.32 & -0.43 & -0.56 & 0.06 & -0.15 & -0.09 & -0.20 \\
\hline & GCF & -0.14 & -0.04 & 0.39 & -0.18 & -0.24 & -0.31 & -0.14 \\
\hline
\end{tabular}

MAX: the maximal absolute change of gingival blood flow (GBF) after the application of light-induced heat; $\mathrm{MAX} \%$ : maximal percentage change relative to the baseline; RT: the time needed to decrease to one third of $\mathrm{MAX} \%$, representing the speed of recovery after hyperaemia; Area: the area under the curve from the start of recording after heat stimulation to the point of RT; GFPA: the average gingival Flux pulse amplitude was calculated at baseline (GFPA-bsl) and the first 15 seconds after heat provocation had been completed (GFPA-heat); $\# p<0.05 ; \# \# p<0.01$

\section{Discussion}

We found that a short application of heat on the human gingiva resulted in a temporary increase in GBF. To the best of our knowledge, there are only two references in the literature on the application of a heat test on the human gingiva. Similarly to our results, after a hot water flush, a hyperaemic response was observed by Baab et al. (3) measured by Laser Doppler Flowmetry and the application of hot water produced a significant increase in pulse amplitude in the healthy gingiva measured by reflection photoplethysmography (20). However, neither the characteristics nor the mechanism of the vascular changes have been described yet.

The mechanism of vasodilation after a heat challenge in the skin is extensively researched $(25,35,48)$. Thermal hyperaemia in the skin is characterized by a biphasic increase in skin blood flow. A rapid initial peak is observed within 1 to 2 minutes after the onset of heat application, which mostly depends on a local sensory nerve axon reflex since it can be significantly attenuated by local anaesthesia (1). The initial peak is followed by a prolonged plateau which mainly depends on the release of nitric oxide $(4,36)$. It should be noted that in our experiment only one peak was observed which may be explained by the short application of heat $(30 \mathrm{~s}$ or $80 \mathrm{~s})$ contrary to skin experiments where it is continuous throughout the measurement whereas, with our technique, recording is not possible during the application of heat. Another difference compared to the skin is the magnitude of the response. In the human skin it is possible to bring about even a 10-fold increase by the application of $42{ }^{\circ} \mathrm{C}$ heat without a pain stimulus, however, based on our results in the human gingiva the increase was less than 2-fold. Subsequent application of light (for a total of $300 \mathrm{~s}$ ) is able to induce a further dose-dependent increase in the GBF (data not shown), but the maximum response was only 3-fold, already accompanied by slight pain in some of the patients. The pain itself could evoke nerve-mediated vasoregulatory alterations which may interfere with heatinduced vascular control mechanisms. Therefore, the pain-induced component of GBF increase has to be avoided during the heat test.

Interestingly, the increase in GBF after heat application was solely due to the increase in the average speed of blood cells without a change in CMBC. By contrast, for the skin, most studies found little or no increase in velocity but did so in $\operatorname{CMBC}(9,60)$. Fredriksson et al. used velocity-resolved quantitative Laser Doppler Flowmetry in the skin and found that 
blood flow, in parallel with CMBC, greatly increased during local heat provocation in the high-velocity region but not in the low-velocity region (10). Based on these findings they concluded that increased flow due to heat provocation is shunted from the artery to the vein side. In the healthy gingival tissue the microvessels supplying the marginal gingiva run perpendicular to the outer surface forming long hairpin capillary loops $(8,39,40)$. They receive their arterial blood supply mainly from the vessels from the periosteum of the alveolar process running parallel to the epithelium. In our experiments the laser Doppler probe was positioned perpendicular to the surface of the gingival margin where Laser Doppler Flowmetry was used to measure the capillary flow of the blood cells moving mostly parallel to the laser beam. We assume that the increase in flow observed in the terminal capillaries was due to proximal arteriolar dilation without opening new capillaries which resulted in an increase in the speed without a change in CMBC. Theoretically, the relatively larger dilation of the arterioles than that of the venules could also contribute to the selectively increased Speed observed. The main function of the increased flow after heat stress is to eliminate excessive local heat, but the metabolic demand of the tissue does not require the opening of inactive capillaries.

The differences in thermal response (magnitude and speed) between the skin and the gingiva may be explained by higher thermal conductance and by the lower basal temperature of the skin. Furthermore, the basal cutaneous blood flow is far less than that of the oral mucosa (22) and this could interpret the higher increase in skin. One of the fundamental roles of skin blood flow control is the thermoregulation of the body. However, the gingiva does not have such a function. It should rather have its own defence mechanisms against heat stress which may be activated during the consumption of hot food or during smoking. Overall, the gingiva seems to have a distinct thermoregulatory mechanism, but further human and animal studies are required to understand the vascular regulatory mechanism in the gingiva subsequent to a thermal challenge.

Heat challenge to the skin is usually applied using a thermostatic heating probe device made of solid metal $(13,41,47)$. But this method is not practical in the case of the gingiva because its surface is curved; in addition, the heating device could mechanically compress the thin gingival tissues including vessels. We used two relatively simple clinically applicable techniques, namely warm saline and light-induced heat provocation. We have found that both methods can be applied on the human gingiva with distinct benefits. Warmed saline is routinely applied in the oral cavity as a daily rinse after tooth extraction to prevent alveolar osteitis (43) and it may improve oedema as well by stimulating lymphatic circulation (52). Based on our experiments, local warming can have additional benefits after oral flap surgery as it may increase blood flow in the low perfused flap, especially after augmentation procedures. However, dropping saline on the gingiva may cause mechanical stimulation on the mucosa which may also interfere with the examination method while the spreading fluid can cause a certain degree of discomfort to the patient and may also interfere with Laser Doppler Flowmetry. Light-induced heat has advantages in experimentation as it involves no mechanical stimulation, causes no discomfort and allows for a better timing of heat application (start and end). Despite similar peak responses light resulted in a prolonged increase in GBF which is possibly due to the deeper penetration of heating light beams and to the longer stimulus.

In this pilot study we also investigated the relationship between blood flow in the marginal gingiva and periodontal inflammation. No correlation was found between periodontal inflammation and GBF at rest. Studies investigating the effect of periodontal 
inflammation on the basal gingival blood flow found conflicting results. Some animal studies $(16,23)$ demonstrated increased blood flow in the inflamed gingiva involving bone loss. However, in the same species, Baab and Öberg (2) found no significant correlation between the gingival index, GCF and blood flow and the elimination of the inflammation did not result in a decrease in blood flow either. In humans, experimentally induced gingivitis resulted in decreased blood flow to the gingiva $(11,33)$ whereas developed gingivitis resulted in increased blood flow (11). GBF at rest was found to be smaller in periodontitis patients compared to the reference subjects (34) and the treatment of gingivitis (58) or periodontitis (15) reduced blood flow. One possible explanation for conflicting results is variations in gingival blood flow as a function of time and the location of the laser Doppler probe. Temporal variation related to biological variation may be influenced by many physiological factors in addition to the inflammation, such as circadian rhythm (54), blood pressure (50), temperature (20) or tooth brushing $(11,45,55)$. Furthermore, although no data are available about the effects of disinfectant mouth rinses, eating and drinking on GBF they may also influence the recordings. That is why we kept all these factors standardized before and during the measurements. To overcome this problem and to better control the temporal and spatial variation of blood flow, a relative functional measurement, the heat provocation test was implemented on the gingiva instead of an absolute one. As a result of the application of heat of standard temperature a relationship was revealed between inflammation and gingival circulation. In contrast to GBF at rest, the MAX value, the GFPA-bsl and the GFPA-heat values were found to be positively correlated to GCF. GBF also returned to the level measured at rest more quickly in the case of more severe inflammation. These findings suggest that the heat provocation test could be a useful tool to detect vascular changes in periodontal inflammation.

Similarly to our results, faster recovery of the blood flow after cooling was found in periodontitis (2) but according to another study (37) the temperature of the gingiva seems to recover slower in periodontitis. These data suggest that the observed shorter vascular response after a thermal challenge does not necessarily mean better thermoregulation; instead, regulation can be impaired as well. As we could not measure the surface temperature of the gingiva during the provocation test we cannot draw a conclusion on whether thermoregulation was changed by the inflammation. However, we found that increased GCF is accompanied by increased absolute MAX GBF which suggests that increased flow may eliminate the excess heat more quickly. It is also possible that the precondition effect of the continuous inflammatory insults may also facilitate gingival vascular responsiveness. Furthermore, in periodontitis there is an increase in vascular density with more dilated and collateral vessels than in the healthy gingiva which is a possible explanation for the higher magnitude of heat-induced hyperaemia and faster recovery in the case of periodontal inflammation $(26,61,62)$.

An increasing number of studies have suggested that cutaneous circulation can serve as a model for generalized microvascular dysfunction $(17,28,35)$ which can be an early sign of disease before the chief symptoms appear $(19,27-29)$. Whether the vasodilatory function of gingival microvessels can be used as a substitute measurement for the small vessel function in the same manner as in the skin model (17) needs further investigation, but our implemented heat provocation test could be a way to test this in human subjects. Our results are in concord with the findings of an animal experiment (53) where both the periodontal inflammation and diabetes per se influenced gingival vascular reactivity; therefore, we need to carefully distinguish between the effects of local inflammation and the systemic conditions. 
Our results have shown that smokers have similar GBF values at rest as non-smokers. Similarly, other studies found no difference in the GBF at rest of non-smoking and smoking periodontitis patients (34). However, smoking cessation improved GBF and restored GCF to the normal non-smoker level in chronic smokers (38) suggesting that smokers may have reduced gingival blood flow. Unfortunately, comparison was not performed with a nonsmoking group. We found similar extent of the vasodilation after heat challenge in gingiva of smokers and non-smokers. This is another difference to the skin where the vasodilation after heat provocation is attenuated in smokers, possible due to the decreased nitric oxide availability in smokers (7). In our study the blood flow recovered slower after heat stress in smokers from the similar MAX values and contrary to the non-smokers heat decreased relative pulse amplitude, furthermore correlation of blood flow parameters with GCF were diminished. However, the lower range of GCF values in our smoking subjects might be the reason for our findings as the GCF could be reduced by smoking per se both in healthy and inflammated periodontal tissue comparing to the respective non-smokers group $(31,46,57)$. This suggests that smoking may suppress the symptoms of inflammation (12). Previous studies $(42,44)$ have showen that the GCF volume serves as an index for the extent of periodontal destruction and the severity of clinical inflammation, but may be not in the smokers. The limitation of the present study is that only GCF measurement was used for assessing the current degree of inflammation, regardless of the classification of the periodontal disease (gingivitis, chronic periodontitis or aggressive periodontitis) and previous treatment, if there was any. Thus according to the present pilot study we cannot conclude that there is a vascular impairment in gingiva in smokers even though we found some differences in vascular parameters. The clinical classifications of the periodontitis tend not to be correlated to tissue and molecular reactions (24) and call for the setup of a new biologically based model for the classification of periodontal disease. Using one of these novel molecular methods to correlate the vascular reactivity to the severity of the disease may answer this question in smokers.

\section{Conclusions}

Local functional heat challenge tests could be useful methods to examine the vascular reactivity of the gingival tissue. The light induced thermal test seems to be more advantageous under clinical conditions than the warm saline induced one. Compared to the skin, the increase in GBF after heat provocation is much smaller and, interestingly, only the increase in the speed of flowing blood cells are responsible for it and not the increase in their concentration. Furthermore, periodontal inflammation promotes increased peak flow and faster restoration after heating in non-smokers, but not in smokers. Our data suggest that moderate periodontal inflammation may facilitate gingival vascular responsiveness which, however, is suppressed by smoking. At the same time, as smoking itself is able to suppress the clinical symptoms of periodontal inflammation (including GCF), further investigations are needed to compare the vascular reactions of non-smokers and smokers with a more detailed biological classification of periodontal disease status based on molecular markers.

\section{Acknowledgements}

This study was conducted with the support of the Research Fund of the Faculty of Dentistry, Semmelweis University, Budapest, Hungary (4030511613, 4030511599) and the Hungarian Scientific Research Fund (OTKA K112364). The authors wish to thank dental students Júlia Németh, Ádám Nagy and Péter Csányi for their assistance in collecting data. 


\section{REFERENCES}

1. Arildsson M, Asker CL, Salerud EG, Stromberg T: Skin capillary appearance and skin microvascular perfusion due to topical application of analgesia cream. Microvasc. Res. 59, 14-23 (2000)

2. Baab DA, Öberg PA: Laser Doppler measurement of gingival blood flow in dogs with increasing and decreasing inflammation. Arch. Oral Biol. 32, 551-555 (1987)

3. Baab DA, Oberg PA, Holloway GA: Gingival blood flow measured with a laser Doppler flowmeter. J. Periodontal Res. 21, 73-85 (1986)

4. Cracowski JL, Minson CT, Salvat-Melis M, Halliwill JR: Methodological issues in the assessment of skin microvascular endothelial function in humans. Trends Pharmacol. Sci. 27, 503-508 (2006)

5. Czukor J: (WHO epidemiologic studies in Hungary in 1985 and 1991). Fogorv. Sz. 87, 223-235 (1994)

6. Doonan RJ, Hausvater A, Scallan C, Mikhailidis DP, Pilote L, Daskalopoulou SS: The effect of smoking on arterial stiffness. Hypertens. Res. 33, 398-410 (2010)

7. Edvinsson ML, Andersson SE, Xu CB, Edvinsson L: Cigarette smoking leads to reduced relaxant responses of the cutaneous microcirculation. Vascular health and risk management 4, 699-704 (2008)

8. Folke LE, Stallard RE: Periodontal microcirculation as revealed by plastic microspheres. J. Periodontal Res. 2, 53-63 (1967)

9. Freccero C, Holmlund F, Bornmyr S, Castenfors J, Johansson AM, Sundkvist G, Svensson H, Wollmer P: Laser Doppler perfusion monitoring of skin blood flow at different depths in finger and arm upon local heating. Microvasc. Res. 66, 183-189 (2003)

10. Fredriksson I, Larsson M, Nystrom FH, Lanne T, Ostgren CJ, Stromberg T: Reduced arteriovenous shunting capacity after local heating and redistribution of baseline skin blood flow in type 2 diabetes assessed with velocity-resolved quantitative laser Doppler flowmetry. Diabetes 59, 1578-1584 (2010)

11. Gleissner C, Kempski O, Peylo S, Glatzel JH, Willershausen B: Local gingival blood flow at healthy and inflamed sites measured by laser Doppler flowmetry. J. Periodontol. 77, 1762-1771 (2006)

12. Hedin CA, Ronquist G, Forsberg O: Cyclic nucleotide content in gingival tissue of smokers and non-smokers. J. Periodontal Res. 16, 337-343 (1981)

13. Heimhalt-El Hamriti M, Schreiver C, Noerenberg A, Scheffler J, Jacoby U, Haffner D, Fischer DC: Impaired skin microcirculation in paediatric patients with type 1 diabetes mellitus. Cardiovasc. Diabetol. 12, 115 (2013)

14. Hermann P, Gera I, Borbely J, Fejerdy P, Madlena M: Periodontal health of an adult population in Hungary: findings of a national survey. J. Clin. Periodontol. 36, 449-457 (2009)

15. Hinrichs JE, Jarzembinski C, Hardie N, Aeppli D: Intrasulcular laser Doppler readings before and after root planing. J. Clin. Periodontol. 22, 817-823 (1995)

16. Hock JM, Kim S: Blood flow in healed and inflamed periodontal tissues of dogs. J. Periodontal Res. 22, 1-5 (1987)

17. Holowatz LA, Thompson-Torgerson CS, Kenney WL: The human cutaneous circulation as a model of generalized microvascular function. J. Appl. Physiol. (1985) 105, 370-372 (2008)

18. Humphrey LL, Fu R, Buckley DI, Freeman M, Helfand M: Periodontal disease and coronary heart disease incidence: a systematic review and meta-analysis. J. Gen. Intern. Med. 23, 2079-2086 (2008)

19. IJzerman R, de Jongh RT, Beijk MA, van Weissenbruch MM, Delemarre-van de Waal HA, Serne EH, Stehouwer $\mathrm{CD}$ : Individuals at increased coronary heart disease risk are characterized by an impaired microvascular function in skin. Eur. J. Clin. Invest. 33, 536-542 (2003)

20. Ikawa M, Ikawa K, Horiuchi H: The effects of thermal and mechanical stimulation on blood flow in healthy and inflamed gingiva in man. Arch. Oral Biol. 43, 127-132 (1998)

21. Johnson GK, Hill M: Cigarette smoking and the periodontal patient. J. Periodontol. 75, 196-209 (2004)

22. Johnson GK, Squier CA, Johnson WT, Todd GL: Blood flow and epithelial thickness in different regions of feline oral mucosa and skin. J. Oral Pathol. 16, 317-321 (1987)

23. Kaplan ML, Jeffcoat MK, Goldhaber P: Blood flow in gingiva and alveolar bone in beagles with periodontal disease. J. Periodontal Res. 17, 384-389 (1982)

24. Kebschull M, Demmer RT, Grun B, Guarnieri P, Pavlidis P, Papapanou PN: Gingival tissue transcriptomes identify distinct periodontitis phenotypes. J. Dent. Res. 93, 459-468 (2014)

25. Kellogg DL, Jr.: In vivo mechanisms of cutaneous vasodilation and vasoconstriction in humans during thermoregulatory challenges. J. Appl. Physiol. (1985) 100, 1709-1718 (2006)

26. Kennedy JE: Effect of inflammation on collateral circulation of the gingiva. J. Periodontal Res. 9, 147-152 (1974) 
27. Khan F, Belch JJ, MacLeod M, Mires G: Changes in endothelial function precede the clinical disease in women in whom preeclampsia develops. Hypertension 46, 1123-1128 (2005)

28. Khan F, Elhadd TA, Greene SA, Belch JJ: Impaired skin microvascular function in children, adolescents, and young adults with type 1 diabetes. Diabetes Care 23, 215-220 (2000)

29. Kruger A, Stewart J, Sahityani R, O’Riordan E, Thompson C, Adler S, Garrick R, Vallance P, Goligorsky MS: Laser Doppler flowmetry detection of endothelial dysfunction in end-stage renal disease patients: correlation with cardiovascular risk. Kidney Int. 70, 157-164 (2006)

30. Kuo LC, Polson AM, Kang T: Associations between periodontal diseases and systemic diseases: a review of the inter-relationships and interactions with diabetes, respiratory diseases, cardiovascular diseases and osteoporosis. Public Health 122, 417-433 (2008)

31. Luthra K, Grover HS, Aggarwal N, Luthra S: Smoking swings of gingival crevicular fluid secretion. Journal of Indian Society of Periodontology 16, 101-103 (2012)

32. Marcenes W, Kassebaum NJ, Bernabe E, Flaxman A, Naghavi M, Lopez A, Murray CJ: Global burden of oral conditions in 1990-2010: a systematic analysis. J. Dent. Res. 92, 592-597 (2013)

33. Matheny JL, Abrams H, Johnson DT, Roth GI: Microcirculatory dynamics in experimental human gingivitis. J. Clin. Periodontol. 20, 578-583 (1993)

34. Mavropoulos A, Brodin P, Rosing CK, Aass AM, Aars H: Gingival blood flow in periodontitis patients before and after periodontal surgery assessed in smokers and non-smokers. J. Periodontol. 78, 1774-1782 (2007)

35. Minson CT: Thermal provocation to evaluate microvascular reactivity in human skin. J. Appl. Physiol. (1985) 109, 1239-1246 (2010)

36. Minson CT, Berry LT, Joyner MJ: Nitric oxide and neurally mediated regulation of skin blood flow during local heating. J. Appl. Physiol. (1985) 91, 1619-1626 (2001)

37. Mormann WH, Bosiger P, Grau P, Scaroni F: The thermodynamic behaviour of labial gingiva in patients with destructive periodontal disease. J. Clin. Periodontol. 12, 477-493 (1985)

38. Morozumi T, Kubota T, Sato T, Okuda K, Yoshie H: Smoking cessation increases gingival blood flow and gingival crevicular fluid. J. Clin. Periodontol. 31, 267-272 (2004)

39. Naumova EA, Dierkes T, Sprang J, Arnold WH: The oral mucosal surface and blood vessels. Head \& Face Medicine 9, 8 (2013)

40. Newman MG, Takei HH, Klokkevold PR, Carranza FA: Carranza's clinical periodontology. In, $11^{\text {th }}$ edn, St. Louis, MO: Saunders Elsevier,, 2012, p. 1 online resource (xlv, 824 p.)

41. Nicotra A, Asahina M, Young TM, Mathias CJ: Heat-provoked skin vasodilatation in innervated and denervated trunk dermatomes in human spinal cord injury. Spinal Cord 44, 222-226 (2006)

42. Oliver RC, Holm-Pederen P, Loe H: The correlation between clinical scoring, exudate measurements and microscopic evaluation of inflammation in the gingiva. J. Periodontol. 40, 201-209 (1969)

43. Osunde OD, Adebola RA, Adeoye JB, Bassey GO: Comparative study of the effect of warm saline mouth rinse on complications after dental extractions. Int. J. Oral Maxillofac. Surg. 43, 649-653 (2014)

44. Perozini C, Chibebe PC, Leao MV, Queiroz Cda S, Pallos D: Gingival crevicular fluid biochemical markers in periodontal disease: a cross-sectional study. Quintessence Int. 41, 877-883 (2010)

45. Perry DA, McDowell J, Goodis HE: Gingival microcirculation response to tooth brushing measured by laser Doppler flowmetry. J. Periodontol. 68, 990-995 (1997)

46. Persson L, Bergstrom J, Gustafsson A, Asman B: Tobacco smoking and gingival neutrophil activity in young adults. J. Clin. Periodontol. 26, 9-13 (1999)

47. Petrofsky J, Alshammari F, Khowailed IA, Rodrigues S, Potnis P, Akerkar S, Shah J, Chung G, Save R: The effect of acute administration of vitamin D on micro vascular endothelial function in Caucasians and South Asian Indians. Medical science monitor. International Medical Journal of Experimental and Clinical Research 19, 641-647 (2013)

48. Roustit M, Cracowski JL: Assessment of endothelial and neurovascular function in human skin microcirculation. Trends Pharmacol. Sci. 34, 373-384 (2013)

49. Sadzeviciene R, Paipaliene P, Zekonis G, Zilinskas J: The influence of microvascular complications caused by diabetes mellitus on the inflammatory pathology of periodontal tissues. Stomatologija / issued by public institution "Odontologijos studija" ... (et al.) 7, 121-124 (2005)

50. Sasano T, Kuriwada S, Sanjo D: Arterial blood pressure regulation of pulpal blood flow as determined by laser Doppler. J. Dent. Res. 68, 791-795 (1989)

51. Scannapieco FA: Periodontal inflammation: from gingivitis to systemic disease? Compend. Contin. Educ. Dent. 25, 16-25 (2004) 
52. Shimotoyodome A, Meguro S, Hase T, Tokimitsu I, Fujimura A, Nozaka Y: Improvement of macromolecular clearance via lymph flow in hamster gingiva by topical warming and massage. J. Periodontal Res. 35, 310-317 (2000)

53. Sugiyama S, Takahashi SS, Tokutomi FA, Yoshida A, Kobayashi K, Yoshino F, Wada-Takahashi S, Toyama T, Watanabe K, Hamada N, Todoki K, Lee MC: Gingival vascular functions are altered in type 2 diabetes mellitus model and/or periodontitis model. Journal of Clinical Biochemistry and Nutrition 51, 108-113 (2012)

54. Svalestad J, Hellem S, Vaagbo G, Irgens A, Thorsen E: Reproducibility of transcutaneous oximetry and laser Doppler flowmetry in facial skin and gingival tissue. Microvasc. Res. 79, 29-33 (2010)

55. Tanaka M, Hanioka T, Kishimoto M, Shizukuishi S: Effect of mechanical toothbrush stimulation on gingival microcirculatory functions in inflamed gingiva of dogs. J. Clin. Periodontol. 25, 561-565 (1998)

56. Tsilibary EC: Microvascular basement membranes in diabetes mellitus. J. Pathol. 200, 537-546 (2003)

57. Tymkiw KD, Thunell DH, Johnson GK, Joly S, Burnell KK, Cavanaugh JE, Brogden KA, Guthmiller JM: Influence of smoking on gingival crevicular fluid cytokines in severe chronic periodontitis. J. Clin. Periodontol. 38, 219-228 (2011)

58. Vag J, Fazekas A: Influence of restorative manipulations on the blood perfusion of human marginal gingiva as measured by laser Doppler flowmetry. J. Oral Rehabil. 29, 52-57 (2002)

59. Vracko R, Benditt EP: Capillary basal lamina thickening. Its relationship to endothelial cell death and replacement. J. Cell Biol. 47, 281-285 (1970)

60. Wipke-Tevis DD, Williams DA: Effect of oral hydration on skin microcirculation in healthy young and midlife and older adults. Wound Repair Regen. 15, 174-185 (2007)

61. Zoellner H, Chapple CC, Hunter N: Microvasculature in gingivitis and chronic periodontitis: disruption of vascular networks with protracted inflammation. Microsc. Res. Tech. 56, 15-31 (2002)

62. Zoellner H, Hunter N: Vascular expansion in chronic periodontitis. J. Oral Pathol. Med. 20, 433-437 (1991) 